\title{
Investigation of the efficacy of digital radiography in the diagnosis of pulp calcification with specific software options
}

\begin{abstract}
Introduction: In recent decades, intraoral digital radiography has been accepted as an alternative for conventional radiography due to its diagnostic qualities like "digital adjustments". Pulp calcification is a prevalent occurrence that makes problem in canal instrumentation and reduces the blood flow. The aim of this study was to investigate the efficacy of digital radiography in the diagnosis of pulp calcification with the use of software options.
\end{abstract}

Materials and methods: One hundred human single-canal premolars were selected. The teeth were inserted in a dry human mandible and each tooth was exposed and the images were interpreted by an experienced oral and maxillofacial radiologist with the help of software options. The images were coded considering the existence of pulp calcification Then the teeth were histologically assessed to verify the existence of pulp calcifications.

Results: The following results were obtained (Table 1).

Radiographic findings: 37 teeth with calcifications and 63 teeth without calcification.

Histopathologic findings: 41 teeth with calcification and 59 teeth without calcification.

Kappa assessment $(\mathrm{P}<0.001)$ and a coefficient of 0.916 showed high agreement between radiologic and pathologic findings (Sensitivity $=90.2 \%$, Specificity $=100 \%$ ).

Discussion: Comparison of digital radiographic images with the gold standard showed that enhanced digital images have high accuracy in the diagnosis of pulp calcifications. In this study we were able to diagnose large numbers of pulp calcifications by using specific software options. It was concluded in the present study that use of Pseudo, Negative, Sharpening and Smoothening software options significantly help diagnose pulp calcifications.

Keywords: pulp calcification, digital radiography, software options
Volume I Issue 4 - 2014

\author{
Mozhdeh Mehdizadeh,' Moeen Hosseini \\ Shirazi, ${ }^{2}$ Foroozan Farahbod, ${ }^{2}$ Razieh Taghavi ${ }^{3}$ \\ 'Department of Oral Radiology, Isfahan University of Medical \\ Sciences, Iran \\ ${ }^{2}$ Department of Operative Dentistry, Isfahan University of \\ Medical Sciences, Iran \\ ${ }^{3}$ Doctor of Dental Surgery, Iran
}

Correspondence: Mozhdeh Mehdizadeh, Department of Oral Radiology, School of Dentistry \& Torabinejad Dental Research Center, Isfahan University of Medical Sciences, Isfahan, Iran, Email mehdizadeh@dnt.mui.ac.ir

Received: September 10, 2014 | Published: September 17, 2014

\section{Introduction}

In recent decades with technological advances and due to limitations in the use of conventional radiography, intraoral digital radiography has been accepted as an alternative for conventional radiography and many researchers believe that digital radiography has most of the required diagnostic qualities compared to conventional radiography.

One of these characteristics is the possibility of making "digital adjustments" using image processing algorithms, which leads to a better display of various structures. Some software programs have different algorithms for "sharpening", "pseudo-coloring", "inverting" and providing a high resolution in order to achieve a better image. ${ }^{1}$ In addition, radiography has always been considered a valuable tool in the diagnosis of pulp diseases and in determining an appropriate treatment plan after clinical diagnosis. ${ }^{2}$ Pulp calcification is a prevalent occurrence (Figure $1 \& 2$ ). Although estimation of the prevalence of such occurrence is very diverse, it is said that pulp calcification occurs in $50 \%$ of teeth and the variation in prevalence is reported between $8 \%$ to $90 \% .^{3,4}$
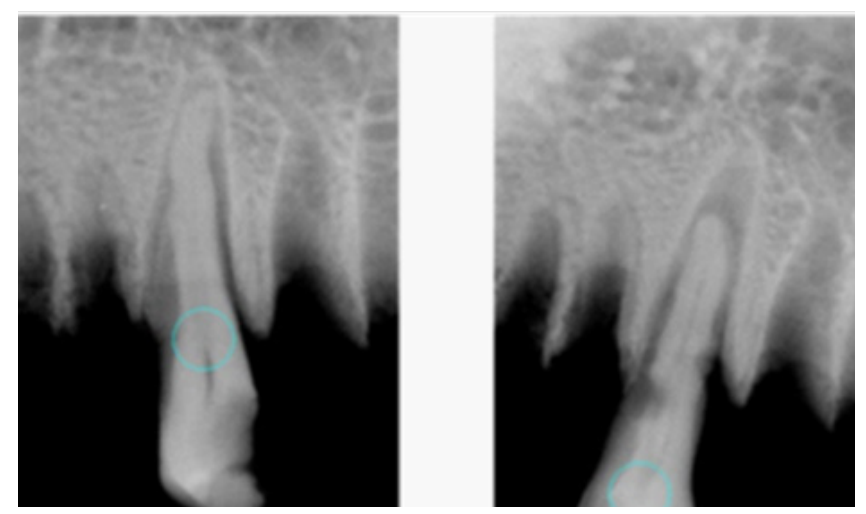

Figure I Pulp calcification radiographic view. 


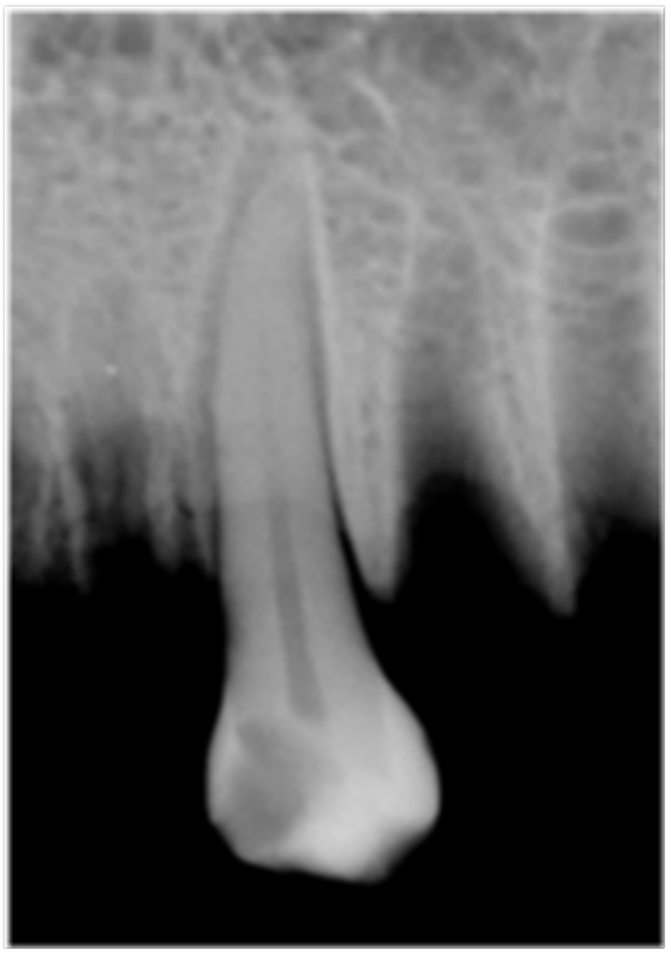

Figure 2 Normal pulp radiographic view.

In the crown pulp, calcification is seen as concentricity called pulp stones. Their prevalence is probably higher in histopathologic assessments because radiographic images can only display pulp stones bigger than $200 \mu \mathrm{m}$ in size. ${ }^{5}$ Radiographic view of pulp stones is very diverse. They may appear as radiopaque structures in root canals and pulp chamber or radiopaque structures that extend from the pulp chamber to the root canal. Conventional radiography is a very useful tool in the diagnosis of pulp stones in pulp chambers. The clinical importance of pulp calcification is the problem they create in canal instrumentation and histologically, calcification replaces the pulp cells, reducing the blood flow. Idiopathic pulpal pain is classically attributed to pulp stones. ${ }^{6}$

It is believed that determination of the presence and size of pulp stones cannot be fully relied on conventional radiographs because these structures have different sizes and calcification grades and cannot be completely detected by conventional radiographs. ${ }^{7}$ Pulp calcifications cannot be clinically diagnosed. ${ }^{6}$ On the other hand, they might be so big that they can be detected on intraoral radiographs as radiopaque structures in canals or pulp chambers, although diffuse calcifications are not detectable on conventional radiographs. ${ }^{8}$

Digital radiography has been used by many dentists all over the world since 1980. Different studies have shown that diagnostic potential of direct systems based on phosphorus plates is higher than that of conventional systems which are based on plain films. ${ }^{3}$ At present, advances in intraoral and extra oral digital radiography with improvements in the use of computer software programs have led to the replacement of conventional radiography by the digital one. ${ }^{4}$

Digital images can be altered by changing the number of pixels and consequently changing gray shadows. In diagnostic imaging, images should be processed in a way that they become compatible with human visual ability. ${ }^{9,10}$ Images with a high number of pixels have high resolution and therefore high quality. ${ }^{4}$ There are various software packages with different algorithms for image processing, which can change contrast, brightness, magnification, pseudo-color, measurement inversion and emboss. ${ }^{9}$

Reddy et al. ${ }^{11}$ evaluated the effect of pseudo-color and contrast of digital radiographs on the diagnosis of Periapical lesions. They concluded that contrast enhancement in digital subtraction radiography technique has a high diagnostic value compared to conventional radiography. Ranjitkar et al. ${ }^{12}$ reported that conventional radiographs can help diagnose pulp stones bigger than $200 \mu \mathrm{m}$ in size and conventional radiography is the safest and the most reliable way of diagnosing pulp stones in clinical studies. However, Hintze ${ }^{13}$ reported that digital radiography has more diagnostic value based on histologic findings as gold standard.

Petrikowski ${ }^{14}$ reported that digital radiography is a very useful alternative for conventional radiography and its popularity is increasing among radiologists due to its high quality. In a study performed by Deva et al., ${ }^{7}$ it was shown that pulp stones and canal calcifications should have a definite size and degree of mineralization to be detected on radiographs. They reported that the size of pulp stones was very diverse and prevalence of pulp stones was higher in bigger pulp chambers.

Goodarzi et al. ${ }^{15}$ compared two computer software programs for root canal length measurements and reported that these two programs have high efficacy based on standard criterion. Mehdizadeh et al. ${ }^{16}$ reported that changing contrast and density is more effective in the diagnosis of Periapical lesions compared to other options. The aim of this study was to investigate the efficacy of digital radiography in the diagnosis of pulp calcification with the use of software options.

\section{Materials and methods}

One hundred human single-canal premolars which had not undergone root canal therapy and fixed prosthetic procedures were selected. The teeth had been extracted due to orthodontic treatments and were stored in formalin solution.

The teeth were inserted in a dry human mandible and each tooth was exposed with $70 \mathrm{kVp}, 10 \mathrm{~mA}$ with CCD sensor for 0.02 seconds. The sensor was fixed to the mandible and was located in the same place for imaging of each tooth. Then images were taken with CCD and saved in Cygnus Media software (Sorodex Helsinki Finland) in a computer with a 13.1-inch Sony LCD monitor. The images were interpreted by an experienced oral and maxillofacial radiologist. All the images were analyzed by the radiologist using the Smoothening, Sharpening, Pseudo and Negative options.

The images were coded considering the existence of pulp calcification:

\section{a. Calcification $=1$ \\ b. No calcifications $=0$}

Then the teeth were sent to the pathology laboratory for histologic assessments to verify the existence of pulp calcifications; $5-\mathrm{mm}$ slices of paraffin blocks were made (Figure 3) and trichrome Masson staining was carried out on the samples. The slices were analyzed for detecting calcifications under an Olympus (CX-2R) light microscope at $\times 100$ and $\times 400$ magnifications by an expert pathologist (Figure 4 and 5). 


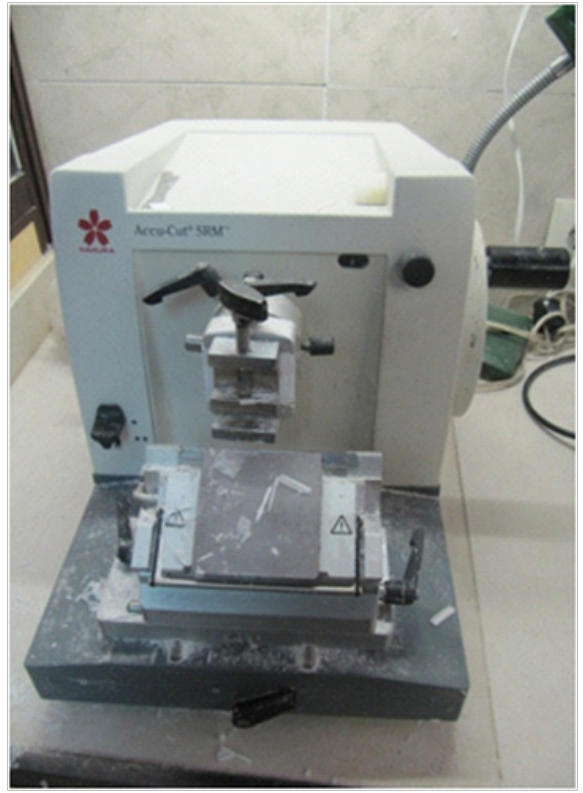

Figure 3 Microtome tool for preparing paraffin slices.

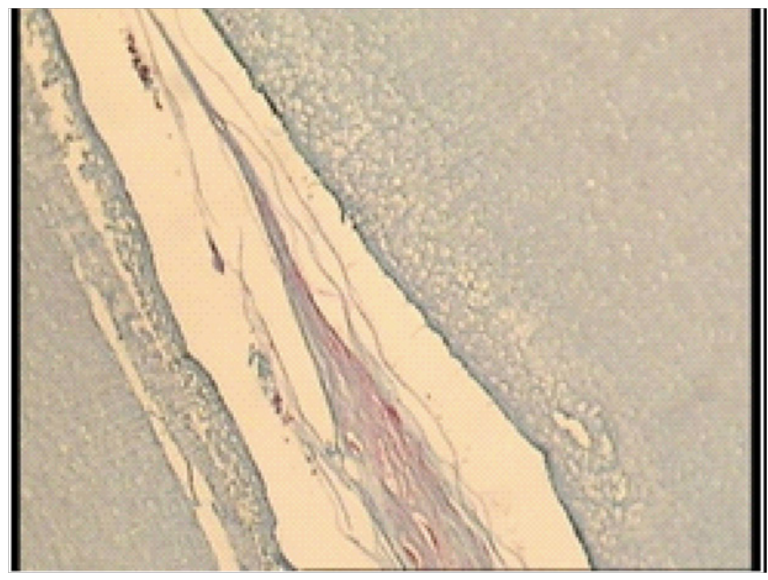

Figure 4 Pulp calcification microscopic view $\times 100$.

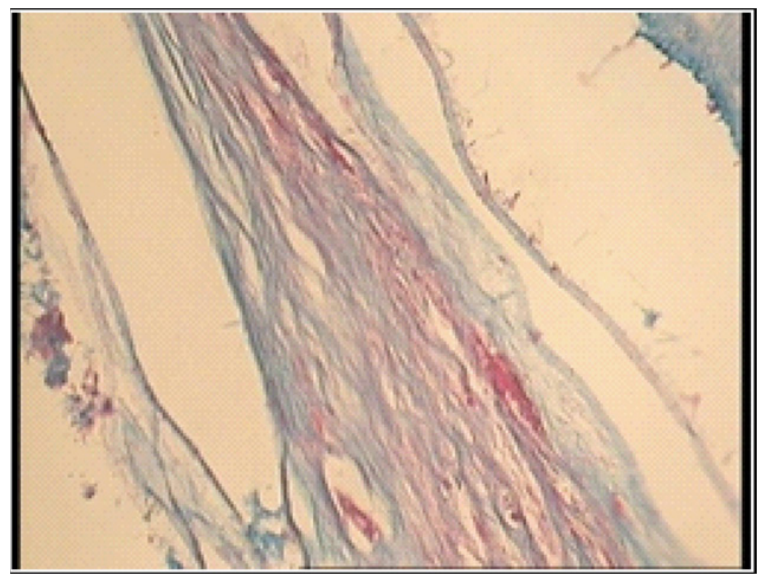

Figure 5 Pulp calcification microscopic view $\times 400$.

\section{Results and discussion}

After collecting the images and tooth slices and their grading these results were obtained (Table 1):

Table I Images and tooth slices and their gradings

\begin{tabular}{lll}
\hline & $\begin{array}{l}\text { Radiological } \\
\text { Findings }\end{array}$ & $\begin{array}{l}\text { Pathological } \\
\text { Findings }\end{array}$ \\
\hline With Clacification & 41 & 37 \\
Without Clacification & 59 & 63
\end{tabular}

a. Radiographic findings: a total of 37 teeth with calcifications and 63 teeth without calcification.

b. Histopathologic findings: a total of 41 teeth with calcification and 59 teeth without calcification.

Kappa assessment $(\mathrm{P}<0.001)$ and a coefficient of 0.916 showed high agreement between radiologic and pathologic findings. Considering the pathologic findings as the gold standard and the assessment of P-value it was concluded that there was significant agreement between the two findings. Kappa agreement coefficient was used for the evaluation of agreement.

$$
\begin{aligned}
& \text { Sensitivity }=\frac{37}{41} \times 100=90.2 \% \\
& \text { Sensitivity }=\frac{59}{59} \times 100=100 \%
\end{aligned}
$$

Radiographic techniques are important and useful tools in different aspects of dentistry. One of the most important uses of radiography is the analysis of dental pulps, for which radiography is the only diagnostic tool. Pulp calcifications have histologic and biologic views similar to dentin, with more calcium content than dentin. Different factors like size and degree of calcification affect the visualization of such structures. ${ }^{7}$ Ranjitkar ${ }^{12}$ evaluated the presence of pulp calcifications by using bitewing radiographs and reported that the majority of these structures were not visible because of superimposition of dentin on conventional radiographs. ${ }^{16}$ In this study, we benefited from digital radiography by increasing the image quality through the use of software options. Comparison of digital radiographic images with the gold standard showed that enhanced digital images have higher accuracy in the diagnosis of pulp calcifications.

Deva et al. ${ }^{7}$ reported that pulp calcifications should have a specific size and calcification degree in order to be visible on Periapical radiographs. However, in this study we were able to diagnose large numbers of pulp calcifications by using specific software options. Eickholz et al. ${ }^{17}$ evaluated the interproximal bone by using enhanced digital radiography and image processing tools like Inversion, High Pass, and Change of Contrast, reporting that changing digital images with the use of basic filters did not lead to better assessment of interproximal structures.

However, by comparison with Deva et al. ${ }^{7}$ article in 2006 about the size and calcification and considering the study of Ranjitkar ${ }^{12}$ in relation to the fact that dentin superimposition reduces the visibility of pulp calcifications, it was concluded in the present study that use of Pseudo, Negative, Sharpening and Smoothening software options significantly help diagnose pulp calcifications. It is possible that Cygnus Media software tool is more efficient in the diagnosis of such structures compared to what Eickholz ${ }^{17}$ used in his study. 
It has been reported previously that use of software tools to increase contrast, reduce noise, reconstruct image and increase details improves the radiographic diagnosis in dentistry and medicine. ${ }^{12}$ With the use of McNemar's test on the results of digital radiography and the use of Cygnus Media to compare the results with the histopathologic findings which were considered as the gold standard, we concluded that digital radiography, with the use of the software programs mentioned above, has high sensitivity and specificity in the diagnosis of pulp calcifications.

\section{Acknowledgments}

None.

\section{Conflict of interest}

The author declares that there is no conflict of interest.

\section{References}

1. Guneri P, Lomcali G, Boyacioglu H, et al. The effects of incremental brightness and contrast adjustments on radiographic data: a quantitative study. Dentomaxillofac Radiol. 2005;34(1):20-27.

2. Naito T, Hosokawa R, Yokota M. Three-dimensional alveolar bone morphology analysis using computed tomography. $J$ Periodontol. 1998;69(50):584-589.

3. Vander SP. Digital radiography as a diagnostic tool in dentistry. Dept of Oral and Maxillofacial Radiology. 2004;11(5):1-6.

4. Brennan J. An introduction to digital radiography in dentistry. J Orthod. 2002;29(1):66-69.

5. Neville B, Damm DD, Allen CM, et al. Oral and Maxillofacial Pathology (3rd edn), Elsevier, Canada, 2009. pp. 984.
6. Stevens C, Berner R. Pathways of the Pulp Expert Consult. 2006.

7. Deva V, Mogoanta L, Manolea H, et al. Radiological and microscopic aspects of the denticles. Rom J Morphol Embryol. 2006;47(3):263-268.

8. White S, Pharoah M. Oral Radiology: Principles and Interpretation. 2013.

9. Langlais RP, Langland OE, Preece J. Princples of Dental Imaging, Lippincott Williams \& Wilkins, USA, 2002. pp. 480.

10. Mol A. Image processing tools for dental applications. Dent Clin North Am. 2000;44(2):299-318.

11. Reddy MS, Bruch JM, Jeffcoat MK, et al. Contrast enhancement as an aid to interpretation in digital subtraction radiography. Oral Surg Oral Med Oral Pathol. 1991;71(6):763-769.

12. Ranjitkar S, Taylor JA, Townsend GC. A radiographic assessment of the prevalence of pulp stones in Australians. Aust Dent J. 2002;47(1):36-40.

13. Hintze H. Diagnostic accuracy of two software modalities for detection of caries lesions in digital radiographs from four dental systems. Dentomaxillofac Radiol. 2006;35(2):78-82.

14. Petrikowski CG. Introducing digital radiography in the dental office: an overview. J Can Dent Assoc. 2005;71(9):651.

15. Goodarzi Pour D, Razmi H, Jabedar Maralani S, et al. New software: comparison between three software programs for root canal length measurement. Dentomaxillofac Radiol. 2008;37(4):228-231.

16. Mehdizadeh M, Karami M, Zamani H. Comparative study of conventional and digital radiography in diagnosis of periapical lesions in dry human mandible. Shiraz Medical University Dentistry. 2008;9(2):163-169.

17. Eickholz P, Riess T, Lenhard $M$, et al. Digital radiography of interproximal bone loss; validity of different filters. J Clin Periodontol. 1999;26(5):294-300. 